\title{
Imaging Hydrated Nanostructured Zeolite X using Single-Electron-Detection Camera
}

\author{
Shaojiang Chen ${ }^{1}$, Don Seo ${ }^{1}$ and Shery L. Y. Chang ${ }^{2}$ \\ 1. School of Molecular Sciences, Arizona State University, Tempe, AZ 85287, United States. \\ 2. LeRoy Eyring Center for Solid State Science, Arizona State University, Tempe, AZ 85287, United \\ States.
}

Zeolites are crystalline microporous aluminosilicate materials that have a three-dimensional framework structure. They have been widely used in catalysis, ion exchange and gas separation due to their high surface area and active sites located inside the micropores [1-3]. However, conventional microsized zeolites suffer from the diffusion limitations due to the very small size of micropores (less than $1 \mathrm{~nm}$ ) and thus limiting their performance. Nanostructured zeolites have demonstrated to be advantageous over the microsized counterparts due the much shorter diffusion path length and high external surface area, which enables the full potential of zeolites to be realized in many applications.

High-resolution TEM has been extensively used to examine the zeolite structures despite the challenges that the zeolite is beam sensitive and can be damaged under the electron beam within a very short time. There have been many successful examples where zeolite can be imaged at very high spatial resolution where the framework is well resolved. However the majority of these examples are (dehydrated) zeolite, or with high $\mathrm{Si} / \mathrm{Al}$ ratio where their electron dose tolerance is significantly higher (more than $10,000 \mathrm{e} / \AA^{2}$ ) [4]. In the case of low $\mathrm{Si} / \mathrm{Al}$ ratio $(<2)$, there is limited success in obtaining high-resolution data so far. It is expected that the dose tolerance can be even lower for the nanostructured zeolites.

Many techniques and treatment have been applied to overcome this problem. For example, using the cooling holder to reduce the specimen damage rate or applying low-dose imaging mode to reduce the total electron dose during TEM imaging. The former suffers from the specimen instabilities/drift due to the bubbling of the cooling holder. The latter suffers from high noise levels arising from both the low electron dose and also the dark noise from the CCD cameras. As a result, the imaging qualities are highly limited.

Here we show that we can overcome this challenge of electron beam damage by imaging at a low dose rate $\left(<5 \mathrm{e}^{-} / \mathrm{A}^{2} / \mathrm{s}\right)$ using a single-electron-detection camera (K2, Gatan Inc). We examined the zeolite $\mathrm{X}$ (faujasite-type framework) nanocrystals with a $\mathrm{Si} / \mathrm{Al}=1.49$ and with a primary particle size around 20 $30 \mathrm{~nm}$. The images were taken using the aberration-corrected TEM (Titan, FEI Company) operated at 300 $\mathrm{kV}$ with a K2 camera operated under "counted mode". Under this mode, the single electron event is directly detected and the DQE of the camera is optimum (at dose rate $<3 \mathrm{e}^{-}$per pixel per second). This capacity is critical in providing good contrast (and good signal to noise ratio) for imaging highly beam sensitive zeolite. Fig. 1 shows a HRTEM image taken at a dose rate about $9 \mathrm{e}$ - $/ \mathrm{pixel} / \mathrm{s}$. Individual frame of 0.2 seconds with a total exposure time of 2 seconds were used and the specimen drifts were measured and corrected before all frames were integrated. It can be seen the final integrated image gives very good contrast despite the low total electron dose.

In addition, we have investigated the relationship between the dose rate and the specimen damage rate. We found that the faujasite particles can sustain more than 300 seconds of continuous imaging without damage if the dose rate is lower than about 5-10 $\mathrm{e}^{-} / \mathrm{A}^{2} / \mathrm{s}$. Moreover, we have also investigated an even 
more beam sensitive Ag-exchanged faujasite particles where the Ag generally reduce into nanoparticles upon electron beam illumination. We found that the $\mathrm{Ag}$ reduction can be reduced when the dose rate is less than $3 \mathrm{e}^{-} / \mathrm{A}^{2} / \mathrm{s}$. Overall, we demonstrate that single-electron-detection cameras provide a solution to imaging highly beam sensitive nanostructured zeolite materials.

\section{References:}

[1] D. W. Breck, Zeolite Molecular Sieves. John Wiley \& Sons, New York, 1974.

[2] A. Corma, Chem. Rev. 6 (1997) p. 2373

[3] V. Valtchev, Chem. Rev. 2013, 113, 6734-6760

[4] R. Csencsits et al, Ultrmicroscopy 23(1987), p. 421.

[5] The authors acknowledge the loan of K2 camera from Gatan, Inc.

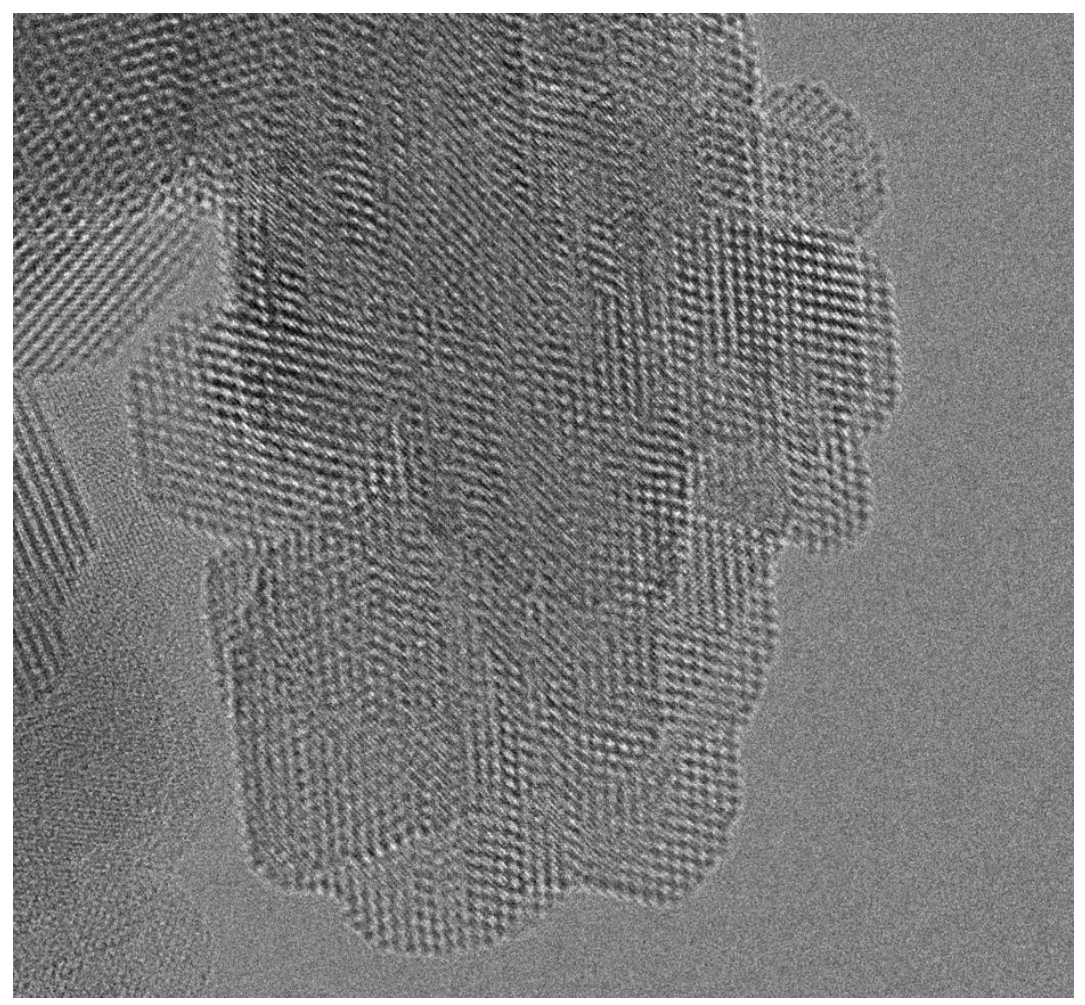

Figure 1. HRTEM images of faujasite particles taken using an aberration-corrected Titan operating at $300 \mathrm{kV}$ equipped with a $\mathrm{K} 2$ single-electron-detection camera. The total dose is approximately 20 electrons per $\AA^{2}$. 